\title{
Charge-induced vortex lattice instability
}

\author{
A. M. Mounce ${ }^{1}$, S. Oh${ }^{1}$, S. Mukhopadhyay ${ }^{1}$, W. P. Halperin ${ }^{1 \star}$, A. P. Reyes ${ }^{2}$, P. L. Kuhns ${ }^{2}$, K. Fujita ${ }^{3}$ \\ M. Ishikado ${ }^{3}$ and S. Uchida ${ }^{3}$
}

It has been predicted that superconducting vortices should be electrically charged and that this effect is particularly enhanced for high-temperature superconductors ${ }^{1,2}$. Hall effect ${ }^{3}$ and nuclear magnetic resonance (NMR) experiments ${ }^{4}$ suggest the existence of charge accumulation in the vortex core, but the effects are small and the interpretation controversial. Here we show that the Abrikosov vortex lattice, characteristic of the mixed state of superconductors, will become unstable at a sufficiently high magnetic field if there is charge trapped on the vortex core. Our NMR measurements of the magnetic fields generated by vortices in $\mathrm{Bi}_{2} \mathrm{Sr}_{2} \mathrm{CaCu}_{2} \mathrm{O}_{8+y}$ single crystals ${ }^{5}$ provide evidence for an electrostatically driven vortex lattice reconstruction with the magnitude of charge on each vortex pancake of $\sim 2 \times 10^{-3} e$, depending on doping, in line with theoretical estimates ${ }^{1,6}$.

The behaviour of quantized vortices in high-temperature superconductors affects many of their applications ranging from high-current-carrying wires ${ }^{7}$ to electronic devices ${ }^{8}$. Effective performance requires that the vortices be stable because their dynamics lead to dissipation originating from the electronic excitations within the vortex cores. Effort to understand the interactions between vortices has been focused on the circulating supercurrents that are characteristic of the vortex and generate local magnetic fields ${ }^{9}$. However, if the vortices are electrically charged there will also be an electrostatic interaction, possibly relevant to vortex stability. We have carried out model calculations showing that if vortices trap electrical charge, then the Abrikosov vortex lattice becomes unstable in sufficiently high magnetic fields where the magnetic interactions diminish and the electrostatics dominate. Our NMR experiments confirm the existence of a magnetic-field-induced instability in the vortex structure of the highly anisotropic superconductor $\mathrm{Bi}_{2} \mathrm{Sr}_{2} \mathrm{CaCu}_{2} \mathrm{O}_{8+y}$ (Bi2212).

A strong magnetic field normal to the conduction plane, $H \| c$, of Bi2212 penetrates in the form of quantized flux bundles, called pancake vortices ${ }^{5}$. They interact magnetically, with a repulsive interaction for pancakes within a plane, but attractive if they lie in different planes. The attractive force is responsible for aligning the vortex cores, one above the other, thereby forming flux lines as indicated in Fig. 1. Perfect alignment is called the Abrikosov configuration. However, the average attractive force decreases ${ }^{9}$ with increasing field as the vortex density increases and as the magnetic field modulations from nearby vortex supercurrents cancel each other more effectively. In contrast, for charged vortex cores, the electrostatic force between pancakes on different planes increases at short range. A complete picture of the balance of these forces requires that the nearby stacks of vortices be taken into account. The calculated cost in magnetic energy for displacing an entire plane of vortices has been calculated, relative to those in all other planes ${ }^{9}$. We follow this approach and compare the magnetic energy with

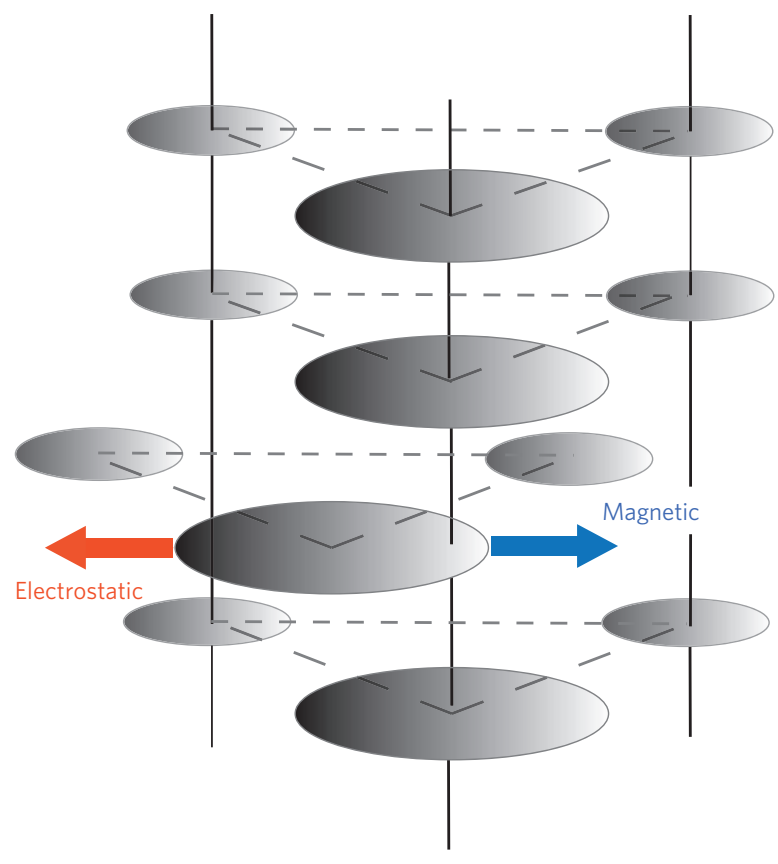

Figure 1 | Vortex pancakes with competing interactions.

Attractive-magnetic and repulsive-electrostatic forces compete and change dominance as the magnetic field is increased. The magnetic interaction between vortices in different planes is attractive preferring a line of vortex pancakes, whereas the Coulomb interaction is repulsive.

the change in electrostatic energy when there is a charge on each vortex core. Such displacements can become more favourable for charged vortices, decreasing the total energy as is illustrated in our calculations shown in Fig. 2. At a sufficiently high magnetic field the electrostatic and magnetic interactions balance and there is an instability. The effect is evident in the inset of this figure at a field of $10 \mathrm{~T}$ for a charge of $2.1 \times 10^{-3} e$, where the energy, as a function of vortex layer displacement in the $a b$ plane, becomes flat.

The existence of charge trapped on the vortex core of a superconductor has been discussed extensively ${ }^{1,2,10-14}$ as a consequence of the Bernoulli potential for the electrostatic fields, and as early as 1961 (ref. 15). Even for conventional superconductors the electrodynamics of vortices lead to a depletion of charge in the vortex core to balance inertial and Lorentz forces ${ }^{10}$. Furthermore, if there is particle-hole asymmetry, it is favourable for charges in the core states to move to a lower energy in the outer superconducting region to maintain a constant chemical potential ${ }^{1,2}$. These different mechanisms produce a vortex charge per pancake ${ }^{1,14}$ of order $Q \sim e\left(\Delta / \epsilon_{\mathrm{F}}\right)^{2}$,

${ }^{1}$ Department of Physics and Astronomy, Northwestern University, Evanston, Illinois 60208, USA, ${ }^{2}$ National High Magnetic Field Laboratory, Tallahassee, Florida 32310, USA, ${ }^{3}$ Department of Physics, University of Tokyo, Tokyo 113-8656, Japan. *e-mail: w-halperin@northwestern.edu. 


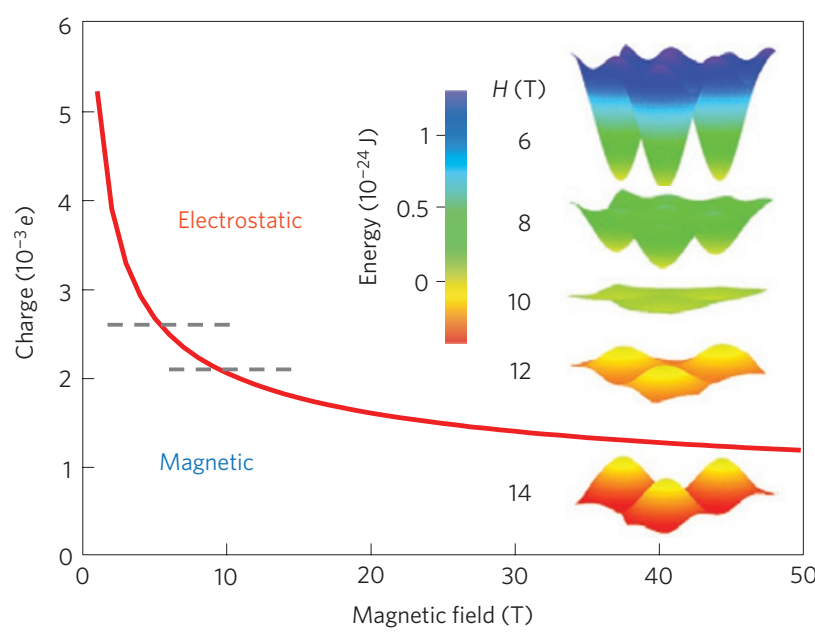

Figure 2 | Energy phase diagram. Two separate regions in the charge-field plane correspond to dominance of charge or magnetic forces. Below the red curve the magnetic energy is larger and above the red curve the electrostatic energy dominates. The dashed lines are chosen to intersect this phase diagram at the magnetic field where the NMR second moments have a minimum (Fig. 3). Inset: At each magnetic field, for a charge of $2.1 \times 10^{-3}$ e, the change in total energy is shown for a rigid displacement of a layer of vortices in the $a b$ plane to various positions ranging over several vortex unit cells of the Abrikosov configuration. Softening of this energy landscape is evident at $10 \mathrm{~T}$.

a reduction from the electronic charge, $e$, by the ratio of the superconducting gap to the Fermi energy squared. This can be of order $10^{-6} e$ for low-temperature superconductors but more favourably, $\sim 10^{-3}$ e for high-temperature superconductors, which have a larger gap. Vortex charging has been studied theoretically for $d$-wave superconductors ${ }^{6,16,17}$ coupled to antiferromagnetic order in the vortex core, and chiral triplet superconductors ${ }^{14}$. The experimental situation is not so well established. A sign change in the Hall effect ${ }^{3}$ has been interpreted ${ }^{1}$ as evidence for vortex charging. However, sign changes have been recently ascribed to Fermi surface effects ${ }^{18}$. Comparison of the nuclear quadrupole resonance frequency and the satellite splittings in NMR has also been interpreted as evidence for vortex charging ${ }^{4}$. However, the magnitude and sign of the charge, and the temperature dependence are in disagreement with the Bardeen-Cooper-Schrieffer theory ${ }^{4,13}$ as well as $d$-wave theory ${ }^{6}$.

We model the competing magnetic and electrostatic interactions by calculating the total energy per vortex for a rigid displacement of a single layer of pancake vortices in the $a b$ plane relative to the energy of the Abrikosov configuration. Josephson coupling between planes is suppressed at a high magnetic field by temperature fluctuations ${ }^{19}$ and can be ignored. The magnetic coupling energy per vortex is given $b^{9}$,

$$
E_{m}(\mathbf{r})=\left(H / \phi_{0}\right) \sum_{\mathbf{g}} U_{0}(\mathbf{g})\left[\mathrm{e}^{i \mathbf{g} \cdot \mathbf{r}}-1\right]
$$

where,

$$
U_{0}(q)=-\phi_{0}^{2} s / 4 \pi \lambda^{4}\left(q^{2}+\lambda^{-2}\right)
$$

Here $H$ is the applied field, normal to the conducting planes; $\mathbf{g}$ is a reciprocal lattice vector of the Abrikosov vortex lattice in $q$-space; $\phi_{0}$ is the flux quantum; $\lambda=220 \mathrm{~nm}$ is the in-plane penetration depth ${ }^{5}$; the bilayer spacing is $s=15 \AA$; and $\mathbf{r}$ is the displacement of the plane of pancakes restricted to the vortex unit cell of the Abrikosov configuration $(\mathbf{r}=0)$. The sums converge when taken to seven times the vortex spacing. For the electrostatic

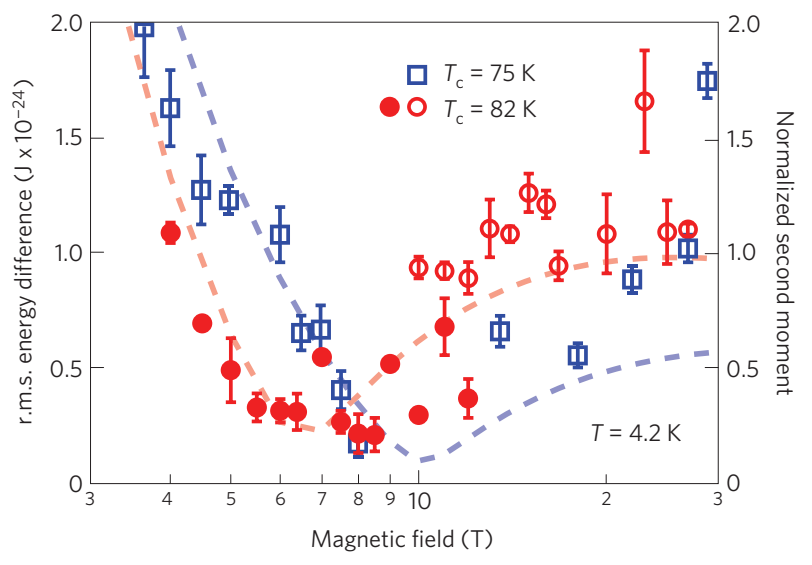

Figure 3 | Softness of the charged vortex structure. The dashed curves are the r.m.s. energy cost for vortex displacements from the Abrikosov configuration perpendicular to the applied field for the effective vortex charges of 2.1 and $2.5 \times 10^{-3}$ e. These curves (left axis) are shown superposed on our NMR data, to demonstrate that the softening of the vortex-vortex interactions for these charges occurs at the magnetic fields where the NMR second moment is a minimum. The second moments of the ${ }^{17} \mathrm{O}$ NMR spectra (right axis) are shown for three crystals independently processed by ${ }^{17} \mathrm{O}$ exchange to give $T_{\mathrm{C}}=75 \mathrm{~K}$ (blue squares) and $T_{\mathrm{C}}=82 \mathrm{~K}$ (red circles, open and filled), at $T=4.2 \mathrm{~K}$ (the error bars represent the background noise). The data are normalized to the theoretical value ${ }^{22}$ for an Abrikosov vortex configuration at low magnetic field, $\sigma_{0}=0.00371 \phi_{0}^{2} / \lambda^{4}$. An independently determined ${ }^{5}$ Knight shift distribution that contributes $1.25 \mathrm{kHz} \mathrm{T}^{-1}$ to the linewidth has been subtracted. At $H=8 \mathrm{~T}$, this corresponds to 0.44 of the normalized second moment.

energy we calculate the Coulomb potential of a periodic lattice and determine the energy required for the displacement defined above. The Coulomb potential for point charges is summed in direct space assuming a cutoff of 35 times the inter-vortex spacing. To maintain charge neutrality, there is a positive background charge spread throughout the unit cell, but when considering the energy difference of a displacement, its effect is cancelled. The calculation of the energy landscape for the displacement of a single plane is shown for various magnetic fields in the inset of Fig. 2. At low magnetic fields the lowest energy corresponds to the Abrikosov configuration where all vortex cores line up, one above the other. However, in high magnetic fields, the most favourable location is for vortices in the displaced plane to line up at the minimum magnetic field position equidistant from vortices in the plane above it (or below it). It is remarkable that at an intermediate field the energy landscape is essentially flat. This condition can be expressed quantitatively in terms of the root-mean-squared (r.m.s.) energy averaged over the unit cell, shown in Fig. 3, and sketched in Fig. 4. The minimum in this r.m.s. energy as a function of magnetic field defines the transition between magnetic and electrostatic order for a given vortex charge. The calculations are carried out for different charges giving the phase diagram in Fig. 2. This calculated charge is really an effective charge that includes the Thomas-Fermi screening within a plane responsible for the suppression factor, $\left(\Delta / \epsilon_{\mathrm{F}}\right)^{2}$, as noted above. However, it does not allow for dielectric screening that will increase the charge by $\sqrt{\kappa}$, where the dielectric constant, $\kappa$, is a materials parameter $\sim 5$ (refs 13,20). As Bi2212 is a bilayer system, the charge per conduction plane in a vortex is larger than the effective charge by $\sqrt{\kappa} / 2 \sim 1$.

The experimental NMR spectrum directly reveals the distribution of magnetic fields generated by vortices and their spatial correlations. In the superconducting state the spectrum is simply proportional to the probability distribution of the local fields. It is 


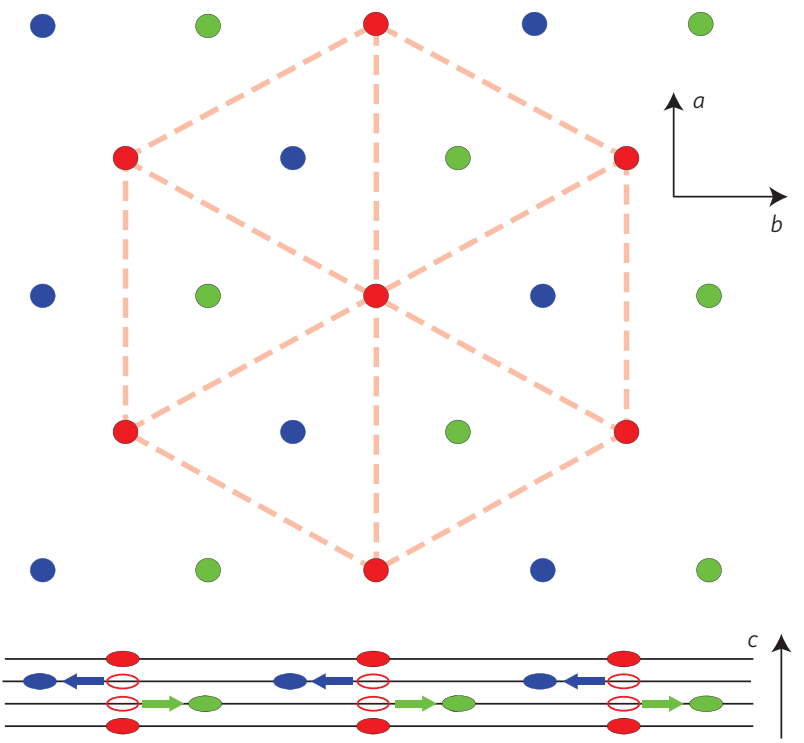

Figure 4 | Electrostatically driven phase change of the vortex lattice. The red symbols, both filled and open, represent vortex pancakes in the Abrikosov configuration. At magnetic fields above the instability, three inequivalent lattices appear (red, blue and green). The aspect ratio in the bottom portion of the figure matches the geometry of $\mathrm{Bi}_{2} \mathrm{Sr}_{2} \mathrm{CaCu}_{2} \mathrm{O}_{8+y}$ at $H=10 \mathrm{~T}$. As the magnetic field increases, the Coulomb potential becomes greater than the magnetic inter-layer coupling, making it favourable for pancake vortices to displace as far as possible from near neighbours in adjacent planes.

common $^{5,21}$ to compare experimental spectra to that expected for a regular array of vortex lines. In this case the field distribution has a characteristic asymmetry and singularities associated with the minimum local field, the saddle point in the field distribution, and a maximum field at the vortex core (inset of Fig. 5). The second moment of the spectrum, closely related to the square of the linewidth, is a direct measure of the inhomogeneity of the fields from vortex supercurrents and can be interpreted in terms of the superconducting penetration length, $\lambda$, and the geometry of the vortex array. If the vortex pancakes are disordered within the conduction plane, the distribution is broadened. In contrast, disorder or reconfiguration of vortices from plane to plane decreases the linewidth ${ }^{22}$. From our NMR experiments we find an unusually abrupt decrease in the linewidth with increasing applied magnetic field for ${ }^{17} \mathrm{O}$ NMR measured at $T=4.2 \mathrm{~K}$ at the $\mathrm{O}(1)$ oxygen site in the copper-oxygen conducting plane of $\mathrm{Bi}_{2} \mathrm{Sr}_{2} \mathrm{CaCu}_{2} \mathrm{O}_{8+y}$. The magnetic field dependence of the second moment of the spectrum is shown in Fig. 3. The magnitude of the second moment at lower fields, $H \lesssim 5 \mathrm{~T}$, is of the order of that expected from an Abrikosov configuration. We interpret the linewidth collapse with increasing field as a transformation to a new, field-induced, vortex state.

The abrupt decrease of the NMR linewidth with increasing magnetic field for several over-doped crystals $\left(T_{\mathrm{c}}=75 \mathrm{~K}, 82 \mathrm{~K}\right.$; Fig. 3) provides consistent experimental evidence that charged vortices can be a mechanism for vortex structure instability. Furthermore, our results indicate that this instability moves to higher magnetic fields with increased doping, corresponding to a smaller vortex charge as suggested by calculations for a $d$-wave superconductor ${ }^{6}$. In related work, it was found that if a single vortex line is charged, it becomes unstable to chiral distortion ${ }^{23}$.

In our model the Abrikosov configuration is transformed to an $A-B-C$-type of tripling of the vortex unit cell along the $c$ axis as sketched in Fig. 4. At high magnetic fields a pancake vortex seeks out a position that is anti-correlated with vortices in planes above and below it. From the minimum NMR linewidth in Fig. 3 we find

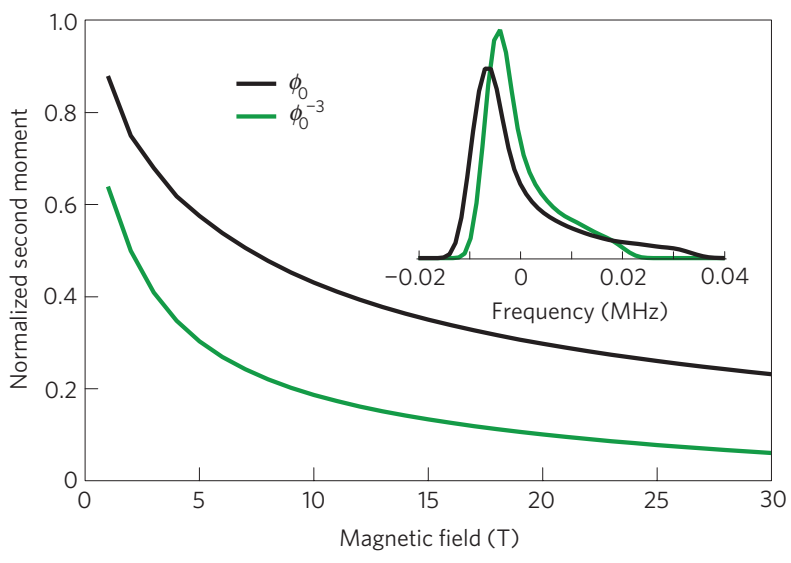

Figure 5 | Magnetic field dependence of the second moment for the vortex local-field distribution. The black curve is calculated from the Ginzburg-Landau theory for the Abrikosov configuration ${ }^{24}$. The green curve corresponds to a tripled vortex unit cell that in our model is an electrostatically driven reconstruction of the vortex lattice obtained by changing $\phi_{0}$ to $\phi_{0} / 3$ as suggested by Brandt ${ }^{22}$ and depicted in Fig. 4. There is a factor of 2-3 between the second moments of the two configurations at any given field. Such a vortex lattice reconstruction leads to a decrease in the second moment similar to our data (Fig. 3). Inset: The calculated NMR spectra at $10 \mathrm{~T}$ for both configurations of vortex pancakes plotted with respect to their first moments.

the magnetic field for this transition. Then according to the phase diagram in Fig. 2, the effective charges per pancake are 2.1 and $2.5 \times$ $10^{-3} \mathrm{e}$ for the crystals $T_{\mathrm{c}}=75 \mathrm{~K}$ and $T_{\mathrm{c}}=82 \mathrm{~K}$, respectively. This new ordering maintains the intra-plane vortex-vortex correlations but the spacing of vortex cores on adjacent planes is decreased by $\sim 1 / \sqrt{3}$. This leads to a decrease in the distribution of local magnetic fields ${ }^{22}$ and a corresponding decrease in the second moment of the NMR spectrum as we show in Fig. 5. Raw spectra and further experimental details are presented in the Supplementary Information. On close examination, the NMR spectra at low fields have two distinct components that merge with increasing field. The composite spectra have an overall shape rather similar to the superposition of the calculated spectra shown in the inset of Fig. 5. We infer that it is likely that the vortex rearrangement proceeds continuously over a range of magnetic fields and completes at the field for which the spectrum width is a minimum. The minimum is a balance between the abruptly decreasing vortex contribution to the local field distribution and an increasing paramagnetic contribution with increasing applied field that we have followed up to as high as $30 \mathrm{~T}$ (Fig. 3). We identify this high-field behaviour with the magnetization induced by bound states in the vortex core ${ }^{21}$.

\section{Methods}

Our samples were prepared by exchanging in 1 bar of flowing ${ }^{17} \mathrm{O}(70-90 \%$ enriched) at $600^{\circ} \mathrm{C}$ for $48 \mathrm{~h}$ followed by annealing for $150 \mathrm{~h}$ at $450^{\circ} \mathrm{C}$. Our $82 \mathrm{~K}$ data in Fig. 3 are from two separate samples from the University of Tokyo. The filled circles correspond to three single crystals of Bi2212, stacked along the $c$ axis, with a total mass of $20 \mathrm{mg}$. The sample represented by the open circles has a similar total mass, although the crystals were cleaved to improve the signal-to-noise ratio. The squares are data from a previous experiment ${ }^{5}$ with crystals, $T_{\mathrm{c}}=75 \mathrm{~K}$, provided by Argonne National Laboratory with similar processing conditions.

NMR measurements were carried out in magnetic fields from 4 to $30 \mathrm{~T}$ at a temperature of $4.2 \mathrm{~K}$ at Northwestern University and the National High Magnetic Field Laboratory in Tallahassee, Florida. We have shown that at this temperature the vortex structure is frozen at all magnetic fields ${ }^{5}$. Spectra were taken using a frequency-sweep technique with a typical $\pi / 2$ pulse of $4 \mu$ s. Before taking data at any particular magnetic field, the sample was heated above $T_{\mathrm{c}}$ for $10 \mathrm{~min}$ and then quench-cooled to $4.2 \mathrm{~K}$. This method produces narrow spectra, with minimal flux pinning, and was found to give consistent results through multiple field cycles. In general, flux pinning is weaker at higher magnetic fields, and if it exists can be identified using field-sweep NMR. 
Received 26 May 2010; accepted 4 October 2010; published online 28 November 2010

\section{References}

1. Khomskii, D. I. \& Freimuth, A. Charged vortices in high temperature superconductors. Phys. Rev. Lett. 75, 1384-1386 (1995).

2. Blatter, G., Feigel'man, M., Geshkenbein, V., Larkin, A. \& van Otterlo, A. Electrostatics of vortices in type-II superconductors. Phys. Rev. Lett. 77, 566-569 (1996).

3. Hagen, S. J., Lobb, C. J., Greene, R. L. \& Eddy, M. Flux-flow Hall effect in superconducting $\mathrm{Tl}_{2} \mathrm{Ba}_{2} \mathrm{CaCu}_{2} \mathrm{O}_{8}$ films. Phys. Rev. B 43, 6246-6248 (1991).

4. Kumagai, K-I., Nozaki, K. \& Matsuda, Y. Charged vortices in high-temperature superconductors probed by NMR. Phys. Rev. B 63, 144502 (2001).

5. Chen, B. et al. Two-dimensional vortices in superconductors. Nature Phys. 3, 239-242 (2007).

6. Knapp, D., Kallin, C., Ghosal, A. \& Mansour, S. Antiferromagnetism and charged vortices in high- $T_{\mathrm{c}}$ superconductors. Phys. Rev. B 71, 064504 (2005).

7. Shen, T. et al. Development of high critical current density in multifilamentary roundwire $\mathrm{Bi}_{2} \mathrm{Sr}_{2} \mathrm{CaCu}_{2} \mathrm{O}_{8+\delta}$ by strong overdoping. Appl. Phys. Lett. 95, 152516 (2009).

8. Martinis, J. M., Ansmann, M. \& Aumentado, J. Energy decay in superconducting Josephson-junction qubits from nonequilibrium quasiparticle excitations. Phys. Rev. Lett. 103, 097002 (2009).

9. Clem, J. R. Two-dimensional vortices in a stack of thin superconducting films: A model for high-temperature superconducting multilayers. Phys. Rev. B 43, 7837-7846 (1991).

10. LeBlanc, M. Vortices in high temperature superconductors: Role of the centrifugal and Lorentz forces. Supercond. Sci. Technol. 10, 919-931 (1997).

11. Koláček, J., Lipavský, P. \& Brandt, E. H. Charge profile in vortices. Phys. Rev. Lett. 86, 312-315 (2001).

12. Šimánek, E. Charged vortices and copper nuclear quadrupole resonance in the cuprates. Phys. Rev. B 65, 184524 (2002).

13. Lipavský, P., Koláček, J., Morawetz, K. \& Brandt, E. H. Electrostatic potential in a superconductor. Phys. Rev. B 65, 144511 (2002).

14. Eschrig, M. \& Sauls, J. A. Charge dynamics of vortex cores in layered chiral triplet superconductors. New J. Phys. 11, 075009 (2009).

15. London, F. Superfluids (Dover, 1961).

16. Chen, Y., Wang, Z. D., Zhu, J-X. \& Ting, C. S. Vortex charges in high-temperature superconductors. Phys. Rev. Lett. 89, 217001 (2002).
17. Zhao, H-W., Zha, G-Q., Zhou, S-P. \& Peeters, F. M. Long-range Coulomb repulsion effect on a charged vortex in high-temperature superconductors with competing $d$-wave and antiferromagnetic orders. Phys. Rev. B 78, 064505 (2008)

18. LeBoeuf, D. et al. Electron pockets in the Fermi surface of hole-doped high- $T_{\mathrm{c}}$ superconductors. Nature 450, 533-536 (2007).

19. Glazman, L. I. \& Koshelev, A. E. Thermal fluctuations and phase transitions in the vortex state of a layered superconductor. Phys. Rev. B 43, 2835-2843 (1991).

20. Maki, M. et al. Dielectric properties and carrier dynamics in $\mathrm{Bi}_{2} \mathrm{Sr}_{2} \mathrm{YCu}_{2} \mathrm{O}_{8+\delta}$. J. Phys. Soc. Jpn 76, 044711 (2007).

21. Mitrović, V. F. et al. Spatially resolved electronic structure inside and outside the vortex cores of a high-temperature superconductor. Nature $\mathbf{4 1 3}$, 501-504 (2001).

22. Brandt, E. H. Magnetic-field variance in layered superconductors. Phys. Rev. Lett. 66, 3213-3216 (1991).

23. Gurevich, A. Helical instability of charged vortices in layered superconductors. Phys. Rev. B 81, 100505 (2010).

24. Brandt, E. H. Precision Ginzburg-Landau solution of ideal vortex lattices for any induction and symmetry. Phys. Rev. Lett. 78, 2208-2211 (1997).

\section{Acknowledgements}

We thank E. H. Brandt, L. N. Bulaevskii, C. A. Collett, M. Eschrig, M. R. Eskildsen, W. J. Gannon, A. Gurevich, A. E. Koshelev, J. Li, V. F. Mitrović, J. Pollanen and J. A. Sauls for helpful discussions. This work was supported by the Department of Energy, contract DE-FG02-05ER46248, and the National High Magnetic Field Laboratory, the National Science Foundation and the State of Florida.

\section{Author contributions}

Experiments were carried out by A.M.M., S.O., S.M., W.P.H., A.P.R. and P.L.K. Samples were provided by K.F., M.I. and S.U.

\section{Additional information}

The authors declare no competing financial interests. Supplementary information accompanies this paper on www.nature.com/naturephysics. Reprints and permissions information is available online at http://npg.nature.com/reprintsandpermissions. Correspondence and requests for materials should be addressed to W.P.H. 